\section{Judicialização no acesso a medicamentos: análise das demandas judiciais no Estado do Rio Grande do Norte, Brasil}

\author{
Judicialization of access to medicines: analysis \\ of lawsuits in the state of Rio Grande do Norte, \\ Brazil
}

\author{
Yonara Monique da Costa Oliveira 1,2 \\ Bárbara Suellen Fonseca Braga 1 \\ Andrezza Duarte Farias 1,2 \\ Cipriano Maia de Vasconcelos 1 \\ Maria Angela Fernandes Ferreira 1
}

doi: 10.1590/0102-311X00174619

\title{
Resumo
}

Este trabalho objetivou analisar os processos judiciais que solicitavam medicamentos ao Estado do Rio Grande do Norte, Brasil, no periodo de 2013 a 2017, descrevendo suas características sociodemográficas, jurídicas e médico-sanitárias. Trata-se de um estudo descritivo, cuja unidade de análise foi o processo judicial individual de solicitação de medicamentos, em que o estado é réu. Os dados foram obtidos por meio de consulta ao sítio eletrônico do Tribunal de Justiça do Estado do Rio Grande do Norte. Foram analisados 987 processos em que a maioria dos autores é do sexo feminino $(58,8 \%)$, com idade média de 48,3 anos e residentes no interior do estado (56,9\%). Houve predomínio de assistência jurídica pública $(52,8 \%)$ e origem da prescrição no setor privado (38,1\%). Em 68\% dos casos, houve acesso ao medicamento, sendo o autor responsável pela compra em $56,1 \%$ deles, via bloqueio de verbas públicas. As doenças crônicas predominaram, bem como a solicitação de medicamentos não disponibilizados pelo Sistema Único de Saúde (SUS). Foram solicitados 1.517 medicamentos, dos quais $936(61,7 \%)$ não estavam na Relação Nacional de Medicamentos Essenciais (Rename). O medicamento mais solicitado foi a insulina glargina (74 pedidos). Dos 10 medicamentos com maior número de ações judiciais, 4 foram posteriormente incorporados ao SUS, com destaque para as insulinas análogas. Os resultados mostraram que a via judicial tem se consolidado como forma de acesso a medicamentos ainda não incorporados ao SUS, o que pode contribuir como forma de pressão para a incorporação. $O$ bloqueio de verbas públicas para o cumprimento das decisões é preocupante para o gestor do SUS, pois compromete a execução das políticas de assistência farmacêutica programadas.

Direito à Saúde; Judicialização da Saúde; Acesso a Medicamentos;

Sistema Único de Saúde

\author{
Correspondência \\ Y. M. C. Oliveira \\ Universidade Federal do Rio Grande do Norte. \\ Av. Senador Salgado Filho 1787, Natal, RN 59056-000, Brasil. \\ yonaraoliveira86@gmail.com \\ 1 Universidade Federal do Rio Grande do Norte, Natal, Brasil. \\ 2 Universidade Federal de Campina Grande, Campina Grande, \\ Brasil.
}




\section{Introdução}

O fenômeno da judicialização da saúde tem se manifestado no Brasil, com maior ênfase, nas duas últimas décadas, principalmente na crescente demanda por medicamentos. As relações entre direito e saúde apresentam grandes desafios para o futuro dos sistemas de saúde, não só no país, mas em grande parte do mundo 1,2 .

As ações judiciais para o fornecimento de medicamentos estão baseadas na garantia do direito à saúde e à vida, conforme estabelecido na Constituição Federal, bem como fundamentadas na Lei no 8.080/1990, que estabelece, no Capítulo I, artigo 6o, a assistência terapêutica integral, inclusive farmacêutica, no campo de atuação do Sistema Único de Saúde (SUS) 3.

Compreender a judicialização da saúde tem sido um esforço empreendido por pesquisadores de todo o Brasil. Na área da Saúde Coletiva, esse tem sido um tema central das pesquisas acadêmicas desde meados dos anos 2000, quando se verificou o crescimento dos casos de judicialização Brasil afora e, consequentemente, de estudos científicos sobre o tema ${ }^{4}$.

Dados oriundos de pesquisas empíricas trazem uma polarização dos argumentos. De um lado, alguns estudos defendem que a judicialização é uma forma legítima dos cidadãos mais vulneráveis economicamente terem acesso a medicamentos e outros bens de saúde que fazem parte do rol de serviços já disponíveis no SUS 5,6,7. Em contrapartida, outros alegam que a judicialização acaba criando uma nova porta de entrada ao SUS, favorecendo indivíduos com maior poder econômico e a própria indústria farmacêutica, ao pressionar pela incorporação de medicamentos ainda não disponíveis no sistema público $8,9,10$.

Os trabalhos que avaliam a demanda judicial por medicamentos são, na sua maioria, descritivos e baseados em análises locais, havendo uma concentração destes nas regiões Sul e Sudeste do país 4,11,12. A ausência de dados nacionais não permite a confirmação de evidências, porém as diferenças encontradas pelos estudos podem revelar a existência de importantes variabilidades regionais no processo de judicialização 13.

Segundo levantamento realizado pelo Conselho Nacional de Justiça (CNJ) sobre o perfil das demandas judiciais na saúde, no período de 2008 a 2017 houve um aumento de 130\% no número de demandas. No mesmo período, mais de 23 mil ações relativas à saúde tramitavam na primeira instância do Tribunal de Justiça do Rio Grande do Norte (TJRN), com uma razão de 76,67 processos a cada 100 mil habitantes, colocando o estado em terceiro lugar no índice de judicialização na área da saúde no país 13 .

Diante do contexto exposto, o objetivo deste trabalho foi analisar as demandas judiciais por medicamentos movidas contra a Secretaria Estadual de Saúde Pública do Rio Grande do Norte (SESAP/ RN) no período de 2013 a 2017.

\section{Metodologia}

Trata-se de um estudo descritivo e retrospectivo, cuja unidade de análise foi o processo judicial individual de solicitação de medicamentos, o qual é movido contra o Estado do Rio Grande do Norte como único réu ou em conjunto com os demais entes públicos.

Foram incluídos na pesquisa processos que tramitavam na 1a instância, com data de distribuição do processo entre janeiro de 2013 e dezembro de 2017. O período de estudo teve como marco inicial o ano da obtenção dos primeiros registros dos processos pela SESAP/RN até o ano de 2017, realizando-se a análise de um quinquênio.

Os dados relativos aos processos judiciais foram inicialmente obtidos junto à Central de Demandas Judiciais (CDJ/SESAP/RN). Nesse banco, fornecido aos pesquisadores, constava o número do processo, o nome do autor, da ação e o bem pleiteado. Com base nessas informações, foi realizada consulta aos autos processuais disponíveis nos sistemas de gerenciamento processuais do TJRN e da Justiça Federal no Rio Grande do Norte (JFRN), a fim de coletar informações detalhadas dos processos e construir o banco com as variáveis de interesse. 
Foram excluídos da análise os processos que tramitavam em segredo de justiça, as ações civis públicas e os que não solicitavam medicamentos. Para a coleta das informações foi construído um instrumento eletrônico semiestruturado, com o uso do programa Microsoft Excel 2013 (https:// products.office.com/).

As variáveis de estudo foram selecionadas baseando-se no Manual de Indicadores de Avaliação e Monitoramento das Demandas Judiciais por Medicamentos 14. As variáveis se enquadraram em três dimensões: (1) sociodemográficas: sexo, idade, ocupação e município de residência do autor da ação; (2) médico-sanitárias: diagnóstico referido nos autos do processo, número de medicamentos solicitados, nome do medicamento solicitado, origem da prescrição médica (serviço público, privado ou filantrópico), presença do medicamento na Relação Nacional de Medicamentos Essenciais (Rename); (3) jurídicas: tipo de representante da ação (público - defensor público e assistência jurídica gratuita ou advocacia privada), data de abertura da ação, decisão liminar e data de sentença; se houve decisão liminar e sentença, forma de obtenção do medicamento (se pelo ente público ou pelo próprio autor da ação) e acesso ao medicamento, atestado pela manifestação nos autos do cumprimento da decisão.

Os dados foram coletados por uma equipe de pesquisa treinada, composta por profissionais da área jurídica e da saúde. Após a coleta, foi realizada a revisão dos dados e a classificação dos medicamentos utilizando o sistema Anatômico e Terapêutico Químico (código ATC). Os diagnósticos foram classificados de acordo com a décima revisão da Classificação Internacional de Doenças (CID-10), sendo usada a categoria diagnóstica principal com base nos dados coletados nos autos processuais. A presença do medicamento nas listas públicas oficiais foi verificada por meio de consulta à Rename 2014, para os processos com data de abertura até a entrada em vigência da Rename 2017. As listas mais recentes, publicadas em 2018 e em 2020, foram consultadas para averiguar a incorporação dos medicamentos mais judicializados. Para a análise descritiva dos dados, utilizou-se o programa SPSS, versão 20 (https://www.ibm.com/). As variáveis contínuas foram categorizadas e apresentadas como frequência absoluta e relativa.

Este trabalho é resultado do projeto de pesquisa intitulado Análise das Demandas Judiciais por Medicamentos no Rio Grande do Norte, que foi submetido e aprovado pelo Comitê de Ética em Pesquisa do Hospital Universitário Onofre Lopes, sob parecer no 2.404.850.

\section{Resultados}

Dos 1.635 processos identificados no período de 2013 a 2017, foram excluídos $138(11,2 \%)$ que tramitavam em segredo de justiça, 81 ações civis públicas $(4,9 \%), 23(1,4 \%)$ processos que solicitavam exclusivamente materiais médico-hospitalares e 361 casos $(22,1 \%)$ que não foram identificados pelo número do processo ou pelo nome da parte nos autos consultados, considerados como perdas. $\mathrm{O}$ universo da pesquisa contou com a análise de 987 processos, dos quais 162 foram do ano de 2013, 217 foram relativos ao ano de 2014, 232 do ano de 2015, 269 de 2016 e 107 processos do ano de 2017.

Quanto às características sociodemográficas, houve prevalência do sexo feminino na autoria das ações (58,8\%), com 75,8\% dos autores acima de 30 anos. A idade média foi de 48,3 anos $( \pm 22,5)$. Quanto à ocupação, foi possível obter esta informação em 663 processos $(67,2 \%)$, sendo a maioria dos autores aposentados $(23,1 \%)$ e estudantes (12,7\%). Dos 167 municípios do estado, foram encontradas demandas de autores residentes em 85 municípios diferentes, a maioria proveniente do interior do estado $(56,9 \%)$ (Tabela 1). Ao considerar a razão do número de ações de cada município para 10 mil habitantes, observou-se uma razão de 16,6 no Município de Caicó, 12,7 em Currais Novos, 5,26 em Açu, 4,6 em Natal e 4,1 em Mossoró (Instituto Brasileiro de Geografia e Estatística. https://cidades. ibge.gov.br/, acessado em 05/Ago/2019).

Quanto à representação jurídica dos autores, observou-se que $52 \%$ foram representados por defensores públicos ou por algum tipo de assessoria jurídica gratuita, contra 47,8\% de advogados privados. Quase 100\% das ações solicitaram uma decisão liminar. Esse pedido foi atendido pelos juízes em $95,6 \%$ dos casos. O tempo mediano decorrido entre a abertura do processo e a concessão das decisões liminares foi de 6 dias (variando de 0 a 868 dias); 78,1\% de tais decisões foram publicadas num período de 0 a 30 dias. A sentença foi proferida em $67 \%$ dos casos (661 processos), com percentual de decisão favorável ao autor de 90,2\% (Tabela 2). 
Tabela 1

Características sociodemográficas dos autores das ações judiciais solicitando medicamentos. Rio Grande do Norte, Brasil, 2013-2017.

\begin{tabular}{|c|c|c|}
\hline Variáveis sociodemográficas & $\mathbf{n}$ & $\%$ \\
\hline \multicolumn{3}{|l|}{ Sexo $(n=987)$} \\
\hline Feminino & 580 & 58,8 \\
\hline Masculino & 407 & 41,2 \\
\hline \multicolumn{3}{|l|}{ Idade $($ anos $)(n=987)$} \\
\hline $0-9$ & 23 & 3,2 \\
\hline $10-19$ & 75 & 10,6 \\
\hline $20-29$ & 74 & 10,4 \\
\hline $30-39$ & 92 & 13,0 \\
\hline $40-49$ & 77 & 10,8 \\
\hline $50-59$ & 103 & 14,5 \\
\hline $60-69$ & 129 & 18,2 \\
\hline 70 ou mais & 137 & 19,3 \\
\hline Ignorado & 277 & 28,0 \\
\hline \multicolumn{3}{|l|}{ Município de residência $(n=987)$} \\
\hline Interior & 562 & 56,9 \\
\hline Capital & 370 & 37,5 \\
\hline Ignorado & 55 & 5,6 \\
\hline \multicolumn{3}{|l|}{ Ocupação (n = 987) } \\
\hline Aposentado(a) & 228 & 23,1 \\
\hline Estudante & 125 & 12,7 \\
\hline Do lar & 62 & 6,3 \\
\hline Desempregado(a) & 29 & 2,9 \\
\hline Agricultor(a) & 27 & 2,7 \\
\hline Beneficiário ou pensionista & 19 & 1,9 \\
\hline Outros & 173 & 17,5 \\
\hline Ignorado & 324 & 32,8 \\
\hline
\end{tabular}

Fonte: elaboração própria a partir dos dados da pesquisa (2019).

Quanto ao acesso ao medicamento pela via judicial, foi possível identificar este desfecho em 788 processos, sendo positivo em $68 \%$ das ações analisadas. O autor da ação foi o responsável pela compra do medicamento em $56,1 \%$ dos casos contra $24,8 \%$ de aquisição pelo ente público.

Foram solicitados 1.517 medicamentos, sendo 328 itens diferentes, com uma média de pedido de $1,55( \pm 1,4)$ medicamento por ação. A maioria dos autores $(74,9 \%)$ solicitou apenas 1 medicamento, $19,4 \%$ (191 processos) solicitaram de 2-3 medicamentos e apenas 5,7\% pediram mais de 3 medicamentos. As prescrições médicas anexadas aos autos dos processos foram provenientes, em sua maioria, de serviços privados de saúde $(38,1 \%)$.

Quanto aos diagnósticos, a categoria mais descrita foi a das doenças endócrinas, nutricionais e metabólicas $(21,2 \%)$, seguida das neoplasias $(15,9 \%)$ e das doenças do aparelho circulatório $(12,1 \%)$. Os medicamentos mais solicitados pertenciam ao grupo dos agentes antineoplásicos e imunomoduladores (21,4\%), seguidos dos medicamentos que atuam no trato alimentar e no metabolismo $(20,2 \%)$, no sistema cardiovascular (13\%) e no sistema nervoso (11,5\%).

Dos 1.517 medicamentos solicitados, 936 (61,7\%) não estavam presentes na Rename. Dos 581 medicamentos incorporados ao SUS, 67,3\% (391) faziam parte do Componente Especializado da Assistência Farmacêutica (CEAF), sujeitos aos critérios de inclusão descritos nos Protocolos Clínicos e Diretrizes Terapêuticas (PCDT) (Tabela 3). 
Tabela 2

Características processuais das ações judiciais solicitando medicamentos. Rio Grande do Norte, Brasil, $2013-2017$.

\begin{tabular}{|c|c|c|}
\hline Variáveis jurídicas & $\mathbf{n}$ & $\%$ \\
\hline \multicolumn{3}{|l|}{ Representante da ação ( $n$ = 987) } \\
\hline Público * & 513 & 52,0 \\
\hline Advogado privado & 472 & 47,8 \\
\hline \multicolumn{3}{|c|}{ Concessão de Antecipação de Tutela ( $n=987$ ) } \\
\hline Sim & 944 & 95,6 \\
\hline Não & 35 & 3,5 \\
\hline Ignorado & 8 & 0,81 \\
\hline \multicolumn{3}{|c|}{ Tempo decorrido até a concessão da antecipação de tutela (dias) $(\mathrm{n}=987)$} \\
\hline $0-30$ & 771 & 78,1 \\
\hline $31-90$ & 151 & 15,3 \\
\hline Mais de 90 & 59 & 6,0 \\
\hline Ignorado & 6 & 0,6 \\
\hline \multicolumn{3}{|l|}{ Sentença proferida $(n=987)$} \\
\hline Sim & 661 & 67 \\
\hline Não & 326 & 33 \\
\hline \multicolumn{3}{|l|}{ Sentença favorável ao autor $(n=661)$} \\
\hline Sim & 596 & 90,2 \\
\hline Não & 57 & 5,8 \\
\hline \multicolumn{3}{|l|}{ Acesso ao medicamento $(n=987)$} \\
\hline Sim & 671 & 68,0 \\
\hline Não & 117 & 11,8 \\
\hline Ignorado & 199 & 20,2 \\
\hline \multicolumn{3}{|c|}{ Forma de obtenção dos medicamentos $(n=987)$} \\
\hline Compra pelo autor & 554 & 56,1 \\
\hline Dispensação pelo ente público & 245 & 24,8 \\
\hline Ambos & 16 & 1,6 \\
\hline Ignorado & 172 & 17,5 \\
\hline
\end{tabular}

Fonte: elaboração própria a partir dos dados da pesquisa (2019).

* Defensoria Pública Estadual ou assistência jurídica gratuita.

O medicamento mais solicitado foi a insulina glargina (74 processos) e, dos 10 medicamentos mais judicializados, apenas 2 (somatropina e rituximabe) estavam incorporados à Rename. Ao analisar esses 10 medicamentos mais demandados, observou-se que 4 foram incluídos na Rename atualmente vigente (2020): insulinas análogas de longa e curta duração e a enoxaparina (Quadro 1).

\section{Discussão}

Após quase duas décadas de experiências com a judicialização da saúde no Brasil, especialmente no acesso a medicamentos, ainda não foi possível traçar um perfil nacional das demandas e dos demandantes. Dados de estudos locais são divergentes em pontos como o perfil socioeconômico dos autores das ações (ricos ou pobres), sobre o bem que requerem (medicamentos novos e caros ou aqueles que deveriam ser disponibilizados por estarem incorporados ao SUS) e sobre a magnitude dos efeitos da judicialização 13. Há uma marcante concentração dos estudos empíricos realizados nas regiões Sul e Sudeste do país, o que não permite extrapolações, principalmente para realidades sociais e econômicas tão distintas, como as que se observam nas regiões Norte e Nordeste ${ }^{12}$. 
Tabela 3

Características médico-sanitárias e de gestão da assistência farmacêutica das ações judiciais por medicamentos. Rio Grande do Norte, Brasil, 2013-2017.

\begin{tabular}{|c|c|c|}
\hline Variáveis médico-sanitárias & $\mathbf{n}$ & $\%$ \\
\hline \multicolumn{3}{|l|}{ Categoria diagnóstica principal (CID-10) $(n=1.102)$} \\
\hline Doenças endócrinas, nutricionais e metabólicas & 234 & 21,2 \\
\hline Neoplasias & 175 & 15,9 \\
\hline Doenças do aparelho circulatório & 133 & 12,1 \\
\hline Doenças do sistema osteomuscular e tecido conjuntivo & 107 & 9,7 \\
\hline Doenças do aparelho respiratório & 72 & 6,5 \\
\hline Doenças do sistema nervoso & 72 & 6,5 \\
\hline Doenças do sangue e órgãos hematopoiéticos & 55 & 5,0 \\
\hline Outros & 254 & 23,1 \\
\hline \multicolumn{3}{|l|}{ Classe terapêutica ATC $(n=1.517)$} \\
\hline L - Agentes antineoplásicos e imunomoduladores & 324 & 21,4 \\
\hline A - Trato alimentar e metabolismo & 307 & 20,2 \\
\hline C - Sistema cardiovascular & 198 & 13,0 \\
\hline $\mathrm{N}$ - Sistema nervoso & 174 & 11,5 \\
\hline H - Preparações hormonais sistêmicas, excluindo insulinas & 140 & 9,2 \\
\hline B - Sangue e órgãos formadores de sangue & 139 & 9,2 \\
\hline $\mathrm{R}$ - Sistema respiratório & 93 & 6,1 \\
\hline Outros & 142 & 9,4 \\
\hline \multicolumn{3}{|l|}{ Origem da prescrição médica $(n=987)$} \\
\hline Serviço privado & 376 & 38,1 \\
\hline Serviço público & 231 & 23,4 \\
\hline Serviço filantrópico & 81 & 8,2 \\
\hline Ignorado & 299 & 30,2 \\
\hline \multicolumn{3}{|l|}{ Presença na Rename $(n=1.517)$} \\
\hline Não & 936 & 61,7 \\
\hline Sim & 581 & 38,3 \\
\hline \multicolumn{3}{|l|}{ Componente da assistência farmacêutica ( $n=581$ ) } \\
\hline CBAF & 190 & 32,7 \\
\hline CEAF & 391 & 67,3 \\
\hline
\end{tabular}

ATC: sistema Anatômico e Terapêutico Químico; CBAF: Componente Básico da Assistência Farmacêutica; CEAF: Componente Especializado da Assistência Farmacêutica; CID-10: Classificação Internacional de Doenças - 10a revisão; Rename: Relação Nacional de Medicamentos Essenciais.

Fonte: elaboração própria a partir dos dados da pesquisa (2019).

A fim de preencher essa lacuna, o presente trabalho analisou processos que solicitavam medicamentos ao Gestor Estadual de Saúde do Rio Grande do Norte. A análise do número de ações por ano mostrou uma tendência de aumento, com o maior número de casos registrados nos anos de $2015 \mathrm{e}$ 2016, e queda no ano de 2017. Ressalta-se que a SESAP/RN não dispõe de um sistema informatizado sobre as demandas judiciais. Os dados eram registrados manualmente pela CDJ, cujo registro informatizado iniciou a partir de uma auditoria realizada pelo Tribunal de Contas da União (TCU) no ano de 2015. Portanto, não é possível afirmar com certeza se houve uma diminuição do número de demandas judiciais por medicamentos no ano de 2017 ou se esta queda ocorreu devido à subnotificação dos registros.

Foi observada a prevalência de mulheres adultas e idosas como autoras das ações. A maior frequência de mulheres em busca de serviços de saúde é corroborada por outros estudos empíricos sobre a judicialização 5,9,15. Já em relação à faixa etária, a maior prevalência de adultas e idosas como requerentes dos pedidos é coerente com a predominância de diagnósticos de doenças crônicas não 


\section{Quadro 1}

Medicamentos mais solicitados nas demandas judiciais de acordo com sua presença na Relação Nacional de Medicamentos Essenciais (Rename). Rio Grande do Norte, Brasil, 2013-2017.

\begin{tabular}{|c|c|c|c|c|}
\hline Medicamento & $\mathbf{n}$ & $\%$ & Presença na Rename 2014 & Presença na Rename 2020 \\
\hline Insulina glargina & 74 & 4,9 & Não & Sim \\
\hline Brometo de tiotrópio & 58 & 3,8 & Não & Não \\
\hline Somatropina & 58 & 3,8 & Sim & Sim \\
\hline Enoxaparina & 57 & 3,7 & Não & Sim \\
\hline Bevacizumabe & 31 & 2,1 & Não & Não \\
\hline Insulina asparte & 31 & 2,1 & Não & Sim \\
\hline Insulina lispro & 30 & 2,0 & Não & Sim \\
\hline Teriparatida & 30 & 2,0 & Não & Não \\
\hline Bevacizumabe intra vítreo & 25 & 1,6 & Não & Não \\
\hline Rituximabe & 25 & 1,6 & Sim & Sim \\
\hline
\end{tabular}

Fonte: elaboração própria a partir dos dados da pesquisa (2019).

transmissíveis, como diabetes mellitus, neoplasias e doenças dos sistemas cardiovascular e respiratório. Os primeiros pedidos judiciais de medicamentos eram de fármacos para o tratamento de doenças infectocontagiosas, especialmente para o tratamento de HIV/aids e hepatites virais. Com a criação de políticas públicas de acesso a esses medicamentos e com o avanço da transição demográfica e epidemiológica da população brasileira, os pedidos foram se diversificando, sendo predominantemente destinados ao tratamento de condições crônicas 16,17.

A ocupação dos autores das ações e a representação jurídica, pública ou privada, são frequentemente utilizadas como indicadores indiretos da situação econômica dos demandantes. Neste estudo, a maioria dos autores foi constituída por aposentados e estudantes e, quanto à representação jurídica, há um discreto predomínio da representação via defensor público ou por outro tipo de assessoria jurídica gratuita. Apesar de não termos acesso a informações sobre renda per capita, o fato de a maioria das ações ter sido representada pela Defensoria Pública Estadual (DPE) leva-nos a supor que pessoas menos favorecidas economicamente estão conseguindo reivindicar na Justiça o acesso a medicamentos, já que são adotados critérios e exigida a comprovação de renda.

Alguns trabalhos realizados em diferentes estados do país, como São Paulo, Minas Gerais, Santa Catarina e Ceará, apresentam predominância de representação jurídica por advogados privados, levantando a tese de que a judicialização é conduzida por indivíduos em menor vulnerabilidade social $8,9,18,19$. Nesse sentido, os nossos achados se assemelham àqueles encontrados em estudos realizados no Rio de Janeiro, Distrito Federal, Rio Grande do Sul e Pará 5,6,7,20,21. Ademais, outra informação pertinente é que a DPE (https://www.defensoria.rn.def.br, acessado em 10/Ago/2019) foi instituída no Rio Grande do Norte no ano de 2003, contando com 13 núcleos de atendimento em todo o estado. Para além das diferenças regionais, a presença de instituições públicas fortes e atuantes, como a DPE, pode estar relacionada a um processo mais intenso de judicialização e, assim, a atuação desta instituição constitui uma importante variável para a compreensão do processo de judicialização 7 .

Houve maior frequência de ações de indivíduos residentes no interior do estado. Resultados semelhantes foram encontrados em pesquisas realizadas no Rio Grande do Sul (92\%) e em Minas Gerais $(63,1 \%) 7,9$. A interiorização da judicialização pode estar relacionada à ampliação da rede de serviços de saúde e jurídicos para cidades menores. No nosso estudo, os 3 municípios com o maior número de ações judiciais para cada 10 mil habitantes foram Caicó, Currais Novos e Açu. Como características 
comuns, esses municípios são os mais desenvolvidos nas suas respectivas microrregiões, inclusive com os melhores Índices de Desenvolvimento Humano (IDH) do estado, sendo de 0,652 para Currais Novos, 0,649 para Caicó e 0,661 na região de Açu. Tais cidades são polos das regiões de saúde, contando com estrutura de hospitais regionais na rede pública e serviços de consultórios privados. Além disso, têm núcleos da DPE, o que viabiliza o acesso à Justiça (DPE. https://www.defensoria.rn.def. br, acessado em 10/Ago/2019). A capital do estado, Natal, apareceu apenas em 4o lugar no índice de judicialização, seguida da segunda maior cidade do estado, Mossoró, em 5o. Uma hipótese que pode justificar esses números é que para os residentes na capital há um programa de mediação oferecido pela DPE, o SUS Mediado, o que pode ter diminuído o número de ações levadas ao Poder Judiciário, resolvendo as demandas de forma administrativa e consensual.

Em relação às variáveis jurídicas, foi vista uma ampla concessão de decisões liminares. Nessas decisões, o juiz se posiciona sem fazer uma avaliação mais profunda do caso, muitas vezes sem a manifestação do réu. Esse achado é um consenso entre os estudos que abordam a judicialização da saúde, com taxas que variam de $88,8 \%$ a $100 \% 12$ e reforça a evidência de que o principal critério judicial para a concessão do benefício é a constatação da necessidade urgente do autor de acesso a determinado medicamento ou procedimento, tendo como respaldo os documentos apresentados pelo próprio demandante, baseados, geralmente, em receituário e laudos médicos 22 . As decisões liminares podem ser revistas ao longo do processo, contudo, nossos achados mostraram que, na maioria das ações que já haviam sido sentenciadas, a decisão liminar foi confirmada. Isso demonstra uma grande fragilidade de defesa do Poder Executivo em conseguir ter seus argumentos acatados, como também é reflexo de um ambiente favorável ao litigante devido à tradição estabelecida pelos juízes brasileiros de interpretar a legislação de forma a garantir amplamente os direitos do cidadão 23.

No presente trabalho, a via judicial se mostrou efetiva para o acesso a medicamentos. Fato interessante e preocupante do ponto de vista da Administração Pública, principalmente num contexto de recursos escassos, foi como aconteceu a obtenção desses medicamentos, em sua maioria adquiridos pelos próprios autores ou diretamente com fornecedores por meio de bloqueios judiciais das contas bancárias estaduais. Em muitas decisões, os juízes definem um prazo exíguo para o cumprimento da liminar ou sentença e, não raro, o réu não consegue cumprir, sendo determinado o bloqueio das contas públicas. O valor é liberado para o autor da ação por intermédio de alvarás judiciais ou transferência direta para farmácias, distribuidoras ou para a própria indústria farmacêutica, com o valor baseado na apresentação de três orçamentos, considerando-se o de menor valor, que em muitos casos é obtido nas páginas eletrônicas de farmácias e distribuidoras de medicamentos.

O sequestro de verbas públicas para o cumprimento de demandas judiciais da saúde vem se tornando uma prática frequente no Judiciário brasileiro, apesar de sua constitucionalidade ser questionada. Há, inclusive, decisões do Supremo Tribunal Federal (STF) limitando essa prática, que afeta as contas públicas, comumente, do ente estatal, inviabilizando a utilização do recurso bloqueado para o uso programado e prejudicando a execução de políticas públicas 24 .

Em relação aos diagnósticos, foi observado neste estudo que os autores das ações sofrem de doenças crônicas. Em concordância com esses diagnósticos, as classes de medicamentos mais solicitadas foram os agentes antineoplásicos e imunomoduladores, medicamentos que atuam no trato alimentar, no metabolismo e no sistema cardiovascular. Pacientes crônicos, com idade mais avançada, frequentemente apresentam várias comorbidades que exigem o uso de medicamentos, geralmente de forma contínua para o seu tratamento. Nesta pesquisa, notou-se que a grande maioria dos demandantes procura o Judiciário para obter apenas 1 medicamento, apesar de em algumas ações terem sido identificados pedidos de até 12 fármacos para um único paciente. Esse dado diverge da maioria dos estudos empíricos, que apresenta médias mais elevadas 7,20, porém semelhante ao estudo realizado em Santa Catarina ${ }^{18}$. Nossa hipótese é que os indivíduos têm usado a via judicial apenas para medicamentos inacessíveis por não estarem incorporados ao SUS.

Em relação à origem da prescrição médica, a maioria delas foi oriunda de serviços privados de saúde, com maior prevalência de prescrição de medicamentos não inclusos na Rename. Não há consenso nos estudos realizados sobre a origem da prescrição e o tipo de medicamento pleiteado. Pesquisas realizadas em Santa Catarina e Minas Gerais 9,18,25,26 apresentaram dados semelhantes aos nossos em relação ao tipo de prescritor, já os estudos realizados nos municípios de São Paulo 15, Rio de Janeiro 16, no Estado de São Paulo 8, no Distrito Federal 6 e no Estado do Ceará 19 registraram 
maior prevalência de prescrições provenientes do serviço público. A falta dessas informações em alguns processos dificultou a análise dos dados, falta esta decorrente da dificuldade no acesso aos processos completos por meio eletrônico. Entretanto, considerou-se os dados disponíveis $(69,8 \%)$ suficientes para atender os objetivos da pesquisa. Sabendo-se que mais de $70 \%$ da população brasileira dependem do SUS 27 , o fato de a maioria das prescrições médicas ter tido origem no serviço privado evidencia que esta população tem um perfil misto, ou seja, consegue ter acesso eventualmente a consultas ou exames no sistema de saúde suplementar, mas busca o SUS para ter acesso aos medicamentos. Cabe ressaltar que, em relação aos serviços filantrópicos, os quais contribuíram com número considerável de pedidos, todos foram provenientes de centros de atendimento oncológico.

Ao analisar a associação entre tipo de representação jurídica e origem da prescrição médica, constatou-se que, dos autores representados pela DPE, 43,7\% obtiveram a prescrição nos serviços do SUS, já 67,6\% dos que foram representados por advogado particular obtiveram a prescrição em serviços de saúde privados. Esse dado exibe uma população com características mista: enquadra-se nos critérios para ser atendida pelas defensorias públicas (como a hipossuficiência), todavia, consegue sua prescrição nos serviços privados. Sabe-se que há grandes vazios assistenciais no SUS, principalmente em relação às consultas com especialistas, o que faz com que os indivíduos busquem por consultas na rede privada de saúde.

Em relação à presença dos medicamentos na Rename, o panorama também é variado, com pedidos de medicamentos predominantemente incorporados ao SUS em alguns estudos realizados no Rio Grande do Sul, nos municípios de São Paulo e Rio de Janeiro, no Pará e no Estado de São Paulo 5,7,15, 17,21,28. Porém, a maioria dos dados empíricos mostra-se semelhante aos nossos resultados, apresentando que os medicamentos mais judicializados não são contemplados na Rename 8,9,18,20,29,30,31. Esses dados evidenciam a variabilidade em alguns aspectos relacionados à judicialização, mesmo quando se analisam estados e cidades de uma mesma região, ou mesmo em diferentes períodos numa mesma região. No entanto, pode-se inferir que a prevalência de prescrições oriundas de serviços eminentemente privados privilegia a prescrição de medicamentos não incorporados ao sistema público de saúde, seja por desconhecimento por parte dos médicos das políticas do SUS, seja por interesses econômicos, como a influência da indústria farmacêutica.

No caso do Rio Grande do Norte, apesar de 2/3 dos medicamentos pleiteados não estarem na Rename, não se pode deixar de observar que uma quantidade considerável está incorporada ao SUS, sendo a maioria do CEAF. Nessa categoria, destacaram-se pedidos dos medicamentos somatropina e rituximabe. A possibilidade de desabastecimento desses medicamentos no fornecimento ou o fato de que a dispensação desses segue regras constantes nos PCDT, e muitos pedidos podem estar em desacordo com essas regras, podem ser justificativas para a judicialização.

A análise dos 10 medicamentos que geraram o maior número de solicitações judiciais corrobora a predominância de fármacos ainda não incorporados ao SUS. No entanto, ao avaliar a presença desses medicamentos na Rename 2020, atualmente em vigência, percebe-se que a maioria dos medicamentos mais judicializados no período do estudo foram incorporados. Trabalhos sobre judicialização têm apontado para os efeitos desse fenômeno na incorporação de tecnologias no SUS. Alguns autores defendem que há atraso na incorporação de medicamentos no sistema público de saúde e, portanto, a judicialização estaria corrigindo deficiências deste processo 7 . Já outros, veem como negativa a influência das demandas judiciais no processo de incorporação de tecnologias no SUS 9.

A pressão gerada pelos processos solicitando medicamentos e procedimentos não incorporados pelo serviço público levou à publicação da Lei no 12.401/2011, que regulamentou e deu mais transparência ao processo de incorporação de tecnologias, instituindo a Comissão Nacional de Avaliação de Tecnologias no SUS (Conitec) como órgão responsável pela avaliação e emissão de pareceres sobre o tema. Apesar de reconhecer os avanços na gestão de tecnologias no SUS, corrigindo possíveis atrasos na incorporação de tecnologias, a Conitec analisa os pedidos sob demanda, podendo ficar susceptível aos interesses do mercado, e não necessariamente às prioridades de saúde da população brasileira. Nesse sentido, fica o questionamento se a pressão pela incorporação via demandas judiciais tem levado à seleção de medicamentos não essenciais, com alternativa terapêutica já incorporada, sem que haja demonstração científica de superioridade e de custo-efetividade 16,29,32.

A insulina glargina foi o medicamento mais solicitado pela via judicial no período analisado, sendo relatada dentre os três medicamentos mais pedidos judicialmente em estudos realizados nos estados 
de Minas Gerais, Santa Catarina e São Paulo 18,33. As insulinas análogas têm sido alvo de polêmica quanto à superioridade em relação às insulinas disponibilizadas no SUS (insulinas humanas NPH e regular). As evidências controversas referentes às vantagens terapêuticas das análogas em relação às insulinas humanas já incorporadas e o alto custo dessas novas tecnologias são razões que explicam a crescente judicialização desses medicamentos 34. Após pareceres negativos de incorporação no ano de 2014, em 2017 as insulinas análogas de ação rápida foram incorporadas ao SUS e, em março de 2019, a insulina glargina também recebeu parecer favorável à incorporação, condicionada ao custo de tratamento ser igual ou inferior ao da insulina NPH 35.

Com relação aos demais medicamentos solicitados, o brometo de tiotrópio, para o tratamento da doença pulmonar obstrutiva crônica (DPOC), e o bevacizumabe, para o tratamento de alguns tipos de câncer, ambos receberam parecer negativo de incorporação pela Conitec, mas têm alternativas terapêuticas na Rename ${ }^{36}$. Já o bevacizumabe para uso intravítreo no tratamento da degeneração macular relacionada à idade (DMRI), recebeu autorização para uso excepcional recentemente, concedida pela Agência Nacional de Vigilância Sanitária (Anvisa), atendendo à solicitação da Conitec, após comprovação de eficácia e segurança por ser também uma alternativa mais custo-efetiva comparada ao ranibizumabe 37 . A teriparatida ainda não teve avaliação pela Conitec, entretanto, existem diversas alternativas terapêuticas disponíveis no SUS indicadas para o tratamento da osteoporose. Por fim, a enoxaparina foi recentemente incorporada, com indicação para o tratamento de trombofilia em gestantes e já se encontra na Rename 2020 36. Pode-se inferir que, pelo menos para dois medicamentos, o bevacizumabe, para o tratamento da DMRI, e a enoxaparina, já amplamente utilizada e com justificativa e protocolo padronizados de uso, a incorporação resolveu um vazio assistencial e contemplou a seleção de um medicamento com evidência de superioridade terapêutica. $\mathrm{O}$ que não parece ser o caso do movimento de incorporação das insulinas análogas.

Um estudo que analisou mais de 50 mil ações contra a Secretaria de Estado da Saúde de São Paulo (SES/SP), entre os anos de 2010 e 2014, e que teve como medicamentos mais solicitados as insulinas glargina e lispro, também exibiu uma dinâmica entre incorporação posterior ao SUS de medicamentos alvo de grande número de ações judicias, mostrando que esta pode estar sendo uma estratégia bem-sucedida da indústria farmacêutica, com a colaboração de prescritores, geralmente dos serviços privados de saúde e de determinados grupos de pacientes para incorporar estes medicamentos ao SUS 38. Em relação às insulinas análogas, o que está no centro do debate é o custo-efetividade de se incorporar uma tecnologia que tem substituto, mas que não mostrou melhoria nos desfechos, nem na qualidade de vida que justifique o preço mais elevado que a tecnologia já disponível no SUS. Para ver o seu impacto financeiro, só no ano de 2016, o Município de Belém (Pará), gastou quase 1 milhão de reais com a aquisição da insulina glargina. Esse impacto financeiro é inclusive justificado pelas secretarias estaduais como um argumento à incorporação, já que a aquisição programada seria mais econômica do que atender às decisões judiciais 39. Pesquisas futuras que avaliem de forma mais aprofundada a relação entre judicialização e incorporação de tecnologias no SUS são necessárias.

Este trabalho tem limitações importantes que devem ser consideradas. O banco de dados foi construído com base em planilhas fornecidas pela SESAP/RN, dados estes que são coletados de forma manual, sem que haja uma sistematização do processo e, portanto, susceptível a sub-registros. Houve um percentual considerável de processos que não puderam ser analisados nos sites do TJRN e do JFRN, pois o número do processo e o nome da parte provavelmente tinham erros na sua digitação, não possibilitando a inclusão destes casos. Outro ponto que limitou o potencial explicativo de algumas variáveis foi a incompletude de alguns dados, marcadamente a ocupação dos autores das ações, a origem da prescrição médica e o acesso ao medicamento pleiteado. O TJRN iniciou seu processo de digitalização em meados de 2014 e, mesmo assim, algumas varas de municípios pequenos do interior continuam a trabalhar com processos físicos, não sendo possível ter acesso a documentos anexados nos autos processuais.

Porém, apesar dessas limitações, conseguiu-se fazer uma análise ampla do processo de judicialização no acesso a medicamentos no Estado do Rio Grande do Norte, com o estudo de um número importante de processos, durante um período de 5 anos. Buscou-se utilizar variáveis descritas em manuais, a fim de contribuir para a comparação com dados empíricos de outros estudos locais e, de tal modo, auxiliar na construção de um panorama mais amplo da judicialização da política pública de medicamentos, especialmente na Região Nordeste do Brasil, onde ainda há uma escassez de estudos. 


\section{Comentários finais}

Os aspectos analisados mostram que o fenômeno da judicialização no Estado do Rio Grande do Norte guarda semelhanças e diferenças com o cenário observado em outros estados. Cidadãos residentes no interior potiguar, representados, em sua maioria, pela DPE, buscaram o Judiciário para obter medicamentos ainda não disponibilizados pelo SUS, destinados ao tratamento de doenças crônicas, prescritos por médicos de serviços privados de saúde.

Assim, a procura da via judicial em busca do direito individual à saúde tem se consolidado como via alternativa de acesso a medicamentos no SUS. Fato importante encontrado neste trabalho foi a forma de obtenção desses medicamentos, que ocorreu via bloqueio de verbas públicas na maioria dos casos. Ao sequestrar o dinheiro das contas do estado, tira-se do gestor a possibilidade de cumprir a política pública programada e, invariavelmente, a parcela da população que não é beneficiada pelas decisões judiciais, pois terá ainda menos dos já escassos recursos da saúde.

Este estudo é inédito no Rio Grande do Norte, apontado em relatório do CNJ como o terceiro colocado no Brasil em relação ao índice de judicialização. Desse modo, esta pesquisa contribui para a melhor compreensão do fenômeno da judicialização no acesso a medicamentos, preenchendo a lacuna de estudos mais amplos na Região Nordeste do Brasil. Também, contribui para a gestão local, uma vez que o controle administrativo das ações judicias na SESAP/RN é insuficiente, não havendo uma rotina de coleta, processamento e análises dos dados que permitam um dimensionamento da judicialização da saúde. Dessa forma, espera-se com este trabalho subsidiar a tomada de decisão dos gestores locais da saúde e ampliar o debate com o Judiciário, com vistas a desenvolver soluções para o problema.

\section{Colaboradores}

Y. M. C. Oliveira e B. S. F. Braga colaboraram no planejamento do estudo, na coleta, análise e interpretação dos dados, redação e revisão crítica do artigo e aprovação da versão final. A. D. Farias e M. A. F. Ferreira colaboraram no planejamento do estudo, análise e interpretação dos dados, redação e revisão crítica do artigo e aprovação da versão final. C. M. Vasconcelos colaborou no planejamento do estudo, na interpretação dos dados, revisão crítica do artigo e aprovação da versão final.

\section{Informações adicionais}

ORCID: Yonara Monique da Costa Oliveira (00000001-7541-7677); Bárbara Suellen Fonseca Braga (0000-0002-6658-9976); Andrezza Duarte Farias (0000-0002-2253-2919); Cipriano Maia de Vasconcelos (0000-0003-0925-5539); Maria Angela Fernandes Ferreira (0000-0002-6142-948X).

\section{Agradecimentos}

Os autores agradecem à Secretaria Estadual de Saúde Pública do Rio Grande do Norte pela disponibilização dos dados para a pesquisa. 


\section{Referências}

1. Sant'Ana JMB, Pepe VLE, Figueiredo TA, Osorio-De-Castro CGS, Ventura M. Racionalidade terapêutica: elementos médico-sanitários nas demandas judiciais de medicamentos. Rev Saúde Pública 2011; 45:714-21.

2. Medici AC. Judicialização, integralidade e financiamento da saúde. Diagn Tratamento 2010; 15:81-7.

3. Conselho Nacional de Secretários de Saúde. Assistência farmacêutica no SUS. Brasília: Conselho Nacional de Secretários de Saúde; 2011.

4. Oliveira MDRM, Delduque MC, Sousa MF, Mendonça AVM. Judicialização da saúde: para onde caminham as produções científicas? Saúde Debate 2015; 39:525-35.

5. Biehl J, Amon JJ, Socal MP, Petryna A. Between the court and the clinic: lawsuits for medicines and the right to health in Brazil. Health Hum Rights 2012; 14:E36-52.

6. Diniz D, Machado TRDC, Penalva J. A judicialização da saúde no Distrito Federal, Brasil. Ciênc Saúde Colet 2014; 19:591-8.

7. Biehl J, Socal MP, Amon JJ. The judicialization of health and the quest for state accountability: evidence from 1,262 lawsuits for access to medicines in southern Brazil. Health Hum Rights 2016; 18:209-20.

8. Chieffi AL, Barata RB. Judicialização da política pública de assistência farmacêutica e eqüidade. Cad Saúde Pública 2009; 25:1839-49.

9. Machado MAA, Acurcio FA, Brandao CMR, Faleiros DR, Guerra Jr. AA, Cherchiglia ML, et al. Judicialization of access to medicines in Minas Gerais state, Southeastern Brazil. Rev Saúde Pública 2011; 45:590-8.

10. Ferraz OLM, Vieira FS. Direito à saúde, recursos escassos e equidade: os riscos da interpretação judicial dominante. Dados Rev Ciênc Sociais 2009; 52:223-51.

11. Leitão LCA, Simões MOS, Simões AEO, Alves BC, Barbosa IC, Pinto MEB. Judicialização da saúde na garantia do acesso ao medicamento. Rev Salud Pública 2014; 16:360-70.

12. Gomes VS, Amador TA. Estudos publicados em periódicos indexados sobre decisões judiciais para acesso a medicamentos no Brasil: uma revisão sistemática. Cad Saúde Pública 2015; 31:451-62.

13. Conselho Nacional de Justiça. Judicialização da saúde no Brasil: perfil das demandas, causas e propostas de solução. Brasília: Conselho Nacional de Justiça; 2019.

14. Pepe VLE, Ventura M, Osorio-de-Castro C, organizadoras. Manual de indicadores de avaliação e monitoramento das demandas judiciais de medicamentos. Rio de Janeiro: Escola Nacional de Saúde Pública Sergio Arouca, Fundação Oswaldo Cruz; 2011.

15. Vieira FS, Zucchi P. Distorções causadas pelas ações judiciais à política de medicamentos no Brasil. Rev Saúde Pública 2007; 41:214-22.
16. Messeder AM, Osorio-de-castro CGS, Luiza VL. Mandados judiciais como ferramenta para garantia do acesso a medicamentos no setor público: a experiência do Estado do Rio de Janeiro, Brasil. Cad Saúde Pública 2005; 21:52534.

17. Borges DCL, Ugá MAD. Conflitos e impasses da judicialização na obtenção de medicamentos: as decisões de 1a instância nas ações individuais contra o Estado do Rio de Janeiro, Brasil, em 2005. Cad Saúde Pública 2010; 26:59-69.

18. Pereira JR, Santos RI, Nascimento Junior JM, Schenkel EP. Análise das demandas judiciais para o fornecimento de medicamentos pela Secretaria de Estado da Saúde de Santa Catarina nos anos de 2003 e 2004. Ciênc Saúde Colet 2010; 15:3551-60.

19. Nunes CFO, Ramos Júnior AN. Judicialização do direito à saúde na Região Nordeste, Brasil: dimensões e desafios. Cad Saúde Colet (Rio J.) 2016; 24:192-9.

20. Pepe VLE, Ventura M, Sant'ana JMB, Figueiredo TA, Souza VDR, Simas L, et al. Caracterização de demandas judiciais de fornecimento de medicamentos "essenciais" no Estado do Rio de Janeiro, Brasil. Cad Saúde Pública 2010; 26:461-71.

21. Oliveira JMCA. A saúde pública jurisdicionalizada no Estado do Pará. http://publicadirei to.com.br/artigos/?cod=eede2d28a4d05ac5 (acessado em 27/Mar/2020).

22. Ventura M, Simas L, Pepe VLE, Schramm FR. Judicialização da saúde, acesso à justiça e à efetividade do direito à saúde. Physis (Rio J.) 2010; 20:77-100.

23. Ferraz OLM. The right to health in the courts of Brazil: worsening health inequities? The Brazilian model of right-to-health litigation. Health Hum Rights 2009; 11:1-12.

24. Ribeiro IV, Costa AV, Sena HGN. A inconstitucionalidade do manejo do sequestro e do bloqueio de contas da Fazenda Pública como mecanismo satisfativo. Cad Ibero Am Direito Sanit 2019; 8:131-51.

25. Campos Neto OH, Acurcio FA, Machado MMA, Ferá F, Barbosa FLV, Cherchiglia ML, et al. Médicos, advogados e indústria farmacêutica na judicialização da saúde em Minas Gerais, Brasil. Rev Saúde Pública 2012; 46:784-90.

26. Mello AF, Soares LSS, Areda CA, Blatt CR, Galato D. Uma abordagem econômica de processos judiciais de medicamentos impetrados contra um município do sul do Brasil. J Bras Econ Saúde 2016; 8:39-46.

27. Stopa SR, Malta DC, Monteiro CN, Szwarcwald CL, Goldbaum M, Cesar CLG. Acesso e uso de serviços de saúde pela população brasileira, Pesquisa Nacional de Saúde 2013. Rev Saúde Pública 2017; 51 Suppl 1:3s. 
28. Marques SB, Dallari SG. Garantia do direito social à assistência farmacêutica no Estado de São Paulo, Brazil. Rev Saúde Pública 2007; 41:101-7.

29. Figueiredo TA, Osorio-de-Castro CGS, Pepe VLE. Evidence-based process for decisionmaking in the analysis of legal demands for medicines in Brazil. Cad Saúde Pública 2013; 29 Suppl:S159-66.

30. Pinto CDBS, Osorio-de-Castro CGS. Gestão da assistência farmacêutica e demandas judiciais em pequenos municípios brasileiros: um estudo em Mato Grosso do Sul. Saúde Debate 2015; 39(n.spe):171-83.

31. Leitão LCA, da Silva PCD, Simões AEO, Barbosa IC, Pinto MEB, Simões MOS. Análise das demandas judiciais para aquisição de medicamentos no Estado da Paraíba. Saúde Soc 2016; 25:801-8.

32. Souza KAO, Souza LEPF, Lisboa ES. Ações judiciais e incorporação de medicamentos ao SUS: a atuação da Conitec. Saúde Debate 2018; 42:837-48.

33. Siqueira PSF. Judicialização em saúde no Estado de São Paulo. In: Direito à Saúde. Brasília: Conselho Nacional de Secretários de Saúde; 2015. (Para Entender a Gestão do SUS - 2015). https://www.conass.org.br/biblioteca/pdf/ colecao2015/CONASS-DIREITO_A_SAUDE -ART_29.pdf.

34. Lisboa ES, Souza LEPF. Por que as pessoas recorrem ao Judiciário para obter o acesso aos medicamentos? O caso das insulinas análogas na Bahia. Ciênc Saúde Colet 2017; 22:1857-64.
35. Secretaria de Ciência, Tecnologia e Insumos Estratégicos, Ministério da Saúde. Portaria no 19, de 27 de março de 2019. Torna pública a decisão de incorporar insulina análoga de ação prolongada para o tratamento de diabetes mellitus tipo I, no âmbito do SUS. Diário Oficial da União 2019; 29 mar.

36. Comissão Nacional de Incorporação de Tecnologias no Sistema Único de Saúde. Recomendações sobre as tecnologias avaliadas. http://conitec.gov.br/decisoes-sobre-incorpo racoes (acessado em 20/Ago/2019).

37. Comissão Nacional de Incorporação de Tecnologias no Sistema Único de Saúde. PCDT sobre degeneração macular é publicado. http://coni tec.gov.br/pcdt-sobre-degeneracao-macular-e - publicado (acessado em 20/Ago/2019).

38. Chieffi AL, Barradas RCB, Golbaum M. Legal access to medications: a threat to Brazil's public health system? BMC Health Serv Res 2017; 17:499.

39. Pinheiro PNQ, Garcia JVM, Carvalho ET, Luz DA, da Silva VG. Farmacoeconomia: gastos com análogos de insulina adquiridos por meio de judicialização em um município do Estado do Pará, Brasil, no ano de 2016. J Bras Econ Saúde 2019; 11:42-8. 


\section{Abstract}

This study aimed to analyze the lawsuits demanding access to medicines in the state of Rio Grande do Norte, Brazil, from 2013 to 2017, describing their sociodemographic, legal, and medi$\mathrm{cal} /$ health characteristics. This was a descriptive study in which the unit of analysis was the individual judicial process for requesting medicines in which the state was the defendant. The data were obtained by consulting the website of the Rio Grande do Norte Court of Justice. A total of 987 suits were analyzed, in which the majority of the plaintiffs were females (58.8\%), with a mean age of 48.3 years, and residing in the state's interior (56.9\%). The legal aid was predominantly public (52.8\%), and $38.1 \%$ of the prescriptions originated in the private healthcare sector. Access to the medicines was obtained in 68\% of the cases, and the plaintiff was responsible for the purchase in $56.1 \%$, via attachment of public funds. Chronic noncommunicable diseases predominated, as did demands for medicines not supplied by the Brazilian Unified National Health System (SUS). A total of 1,517 medicines were requested, of which 936 (61.7\%) were not on the National List of Essential Medicines (Rename). The most frequently demanded drug was insulin glargine (74 case). Of the 10 medicines with the most lawsuits, four were later incorporated by the SUS, featuring insulin analogues. The results showed that legal recourse has been consolidated as a form of access to medicines that have still not been incorporated by the SUS, which can contribute as a form of pressure for such incorporation. The attachment of public funds to comply with these rulings is worrisome for the administration of the SUS, since it jeopardizes the execution of regularly scheduled and budgeted policies in pharmaceutical assistance.

Right to Health; Health's Judicialization; Access to Medications; Unified Health System

\section{Resumen}

El objetivo de este estudio fue analizar los procesos judiciales que solicitaban medicamentos al estado de Rio Grande do Norte, Brasil, durante el periodo de 2013 a 2017, describiendo sus características sociodemográficas, jurídicas y médico-sanitarias. Se trata de un estudio descriptivo, cuya unidad de análisis fue el proceso judicial individual de solicitud de medicamentos, en el que el estado es el acusado. Los datos se obtuvieron a través de la consulta a la página web del Tribunal de Justicia del Rio Grande do Norte. Se analizaron 987 procesos judiciales en los que la mayoría de los autores son del sexo femenino (58,8\%), con una edad media de 48,3 años y residentes en el interior del estado (56,9\%). Hubo un predominio de asistencia jurídica pública $(52,8 \%)$ y origen de la prescripción en el sector privado (38,1\%). En el 68\% de los casos, hubo acceso al medicamento, siendo el autor responsable de la compra en un 56,1\% de ellos, vía bloqueo de fondos públicos. Las enfermedades crónicas predominaron, así como la solicitud de medicamentos no puestos a disposición de los pacientes por parte del Sistema Único de Salud brasileño (SUS). Se solicitaron 1517 medicamentos, de los cuales $936(61,7 \%)$ no se encontraban en la Relación Nacional de Medicamentos Esenciales (Rename). El medicamento más solicitado fue la Insulina glargina (74 pedidos). De los 10 medicamentos con mayor número de acciones judiciales, 4 fueron posteriormente incorporados al SUS, destacándose las insulinas análogas. Los resultados mostraron que la vía judicial se ha consolidado como forma de acceso a medicamentos todavía no incorporados al SUS, lo que puede contribuir como forma de presión para su incorporación. El bloqueo de fondos públicos para el cumplimiento de las decisiones es preocupante para el gestor del SUS, puesto que compromete la ejecución de las politicas de asistencia farmacéutica programadas.

Derecho a la Salud; Judicialización de la Salud; Acceso a Medicamentos; Sistema Único de Salud

Recebido em 09/Set/2019

Versão final reapresentada em 01/Jun/2020

Aprovado em 12/Jun/2020 